\title{
Un discours prérévolutionnaire à l'Académie des sciences : L'exemple de Montalembert
}

\section{Jânis Langins}

\section{(2) OpenEdition \\ Journals}

Édition électronique

URL : https://journals.openedition.org/ahrf/159

DOI : $10.4000 /$ ahrf.159

ISSN : 1952-403X

Éditeur :

Armand Colin, Société des études robespierristes

Édition imprimée

Date de publication : 1 juin 2000

Pagination : 159-171

ISSN : 0003-4436

Référence électronique

Jânis Langins, « Un discours prérévolutionnaire à l'Académie des sciences : L'exemple de Montalembert », Annales historiques de la Révolution française [En ligne], 320 | avril-juin 2000, mis en ligne le 23 janvier 2006, consulté le 23 avril 2022. URL : http://journals.openedition.org/ahrf/159 ; DOl : https://doi.org/10.4000/ahrf.159

Ce document a été généré automatiquement le 23 avril 2022.

Tous droits réservés 


\title{
Un discours prérévolutionnaire à l'Académie des sciences : L'exemple de Montalembert
}

\author{
Jânis Langins
}

1 Les multiples attaques contre l'Académie des sciences pendant la Révolution sont venues essentiellement $\mathrm{du}$ dehors ${ }^{1}$. Face à ces attaques extérieures, une solidarité certaine réunissait les membres de la compagnie. Il est vrai qu'il existait pourtant des lignes de rupture correspondant aux opinions politiques et aux attitudes sociales aussi bien qu'aux rivalités personnelles, et des clans de protection existaient. Mais le chimiste révolutionnaire Fourcroy ou l'astronome royaliste Cassini, si différents qu'ils fussent dans tant de domaines, s'accordaient en général sur le fait que l'Académie méritait une place d'honneur, sous n'importe quel régime politique - soit la République française soit la monarchie. La République des Sciences était quelque chose de réel qui planait au-dessus des régimes politiques, méritait leur protection et le respect de toute société qui se prétendait civilisée. Plus tard, Fourcroy allait manier avec adresse le thème du «vandalisme révolutionnaire » (avec les sous-entendus évidents de barbarie) pour attaquer les vaincus de Thermidor et avancer un programme de création de nouvelles institutions éducatives².

2 L'Académie avait déjà été attaquée sous l'Ancien Régime dans les affaires célèbres de Marat et de Mesmer. Vers la fin des années 1770 et le début des années 1780, elle avait rejeté fermement les prétentions de Mesmer et Marat à avoir découvert des nouvelles sciences. Les partisans de ces derniers avaient attaqué les académiciens officiels, avant tout comme de mauvais savants, incapables de se libérer des idées reçues et des dogmes de la science newtonienne. Mais on les avait aussi attaqués comme membres de corps privilégiés hostiles à toute nouveauté qui ne venait pas d'eux et comme des personnes dont le premier mobile était la défense de leurs propres avantages matériels et de leur prestige personnel. L'avancement désintéressé des sciences n'avait aucune place dans leur activité, sinon comme argument rituel mis en avant hypocritement pour justifier leur existence. 
Plus encore que pendant la Révolution, les académiciens avaient alors maintenu leur solidarité dans la chasse aux charlatans. Pour Charles Gillispie, cette allergie aux charlatans à cette époque est une indication de la naissance d'une véritable profession de savant, qui réserve à ses seuls membres le droit d'attribuer des titres et des places rémunérées, de contrôler l'entrée en son sein, et de juger de la qualité des travaux de ses membres ou de tous ceux qui prétendent au statut de savant ${ }^{3}$. Dans l'affaire Mesmer, par exemple, s'il y eut des savants, comme Jussieu, pour admettre que ses idées avaient quelque valeur, nul parmi les académiciens ne mit en question le droit de la compagnie de prononcer sur la valeur ou le manque de valeur des prétendants à ce statut $^{4}$. Parmi ceux qui critiquaient l'attitude de l'Académie, certains avaient une place éminente et respectable dans la société, tel le marquis de Puységur. Ce ne furent pas seulement les Brissot et les pamphlétaires qui montèrent à l'assaut de l'Académie. Mais ce qui les unissait tous, c'était leur extériorité à l'institution.

Dans cette communication, je vais présenter l'exemple d'une exception à cette observation. Je décrirai le cas d'un membre de l'Académie qui, fort respectueux envers ses confrères au début, est devenu de plus en plus critique quand l'Académie n'a pas voulu entériner ses théories par une approbation formelle.

5 Il s'agit du marquis Marc-René de Montalembert (1714-1800). Non seulement ce membre de la noblesse jouissant de protections puissantes, comme celles du prince de Conti et de Madame de Pompadour, joua, comme diplomate et soldat, un rôle non sans éclat pendant la guerre de Sept Ans à la Cour de Saint-Pétersbourg et de Stockholm, mais il fut aussi associé libre de l'Académie royale des sciences, à partir de 1747, et membre de l'Académie impériale de Saint-Pétersbourg, avant même que Buffon et d'Alembert aient eu cet honneur. S'étant lancé d'abord dans la métallurgie, au lendemain de la guerre de Succession d'Autriche, il s'est tourné vers la fortification après sa faillite dans cette entreprise. En 1761, il écrivit à Choiseul, ministre de la Guerre, lui proposant un système totalement nouveau de fortification qui se distinguait par l'absence de bastions et par un nombre sans précédent de feux couverts 5 . Au début, il appela son système La Fortification perpendiculaire. Les ingénieurs auxquels le ministre demanda de se prononcer sur le nouveau système, se montrèrent circonspects et même sceptiques. Parmi eux se trouvait Charles-René Fourcroy de Ramecourt, qui allait devenir quelques années plus tard le véritable chef du Corps royal du génie ${ }^{6}$. Le désaveu de Vauban par Montalembert était trop difficile à accepter, car Vauban était non seulement le héros et le modèle du corps mais aussi l'auteur de sa méthode d'architecture militaire. Montalembert fut donc remercié et prié de garder le secret sur son système pour le bien du pays.

6 Il continua cependant à travailler sur son système et à assiéger les bureaux de divers ministères, où il affronta encore des ingénieurs militaires (y compris Fourcroy) au sujet de projets de fortification coloniale. Est-ce le changement de régime à la mort de Louis $\mathrm{XV}$ et le retour au pouvoir de Maurepas qui amenèrent Montalembert à renoncer à l'injonction au silence de Choiseul ? Est-ce le triomphe final, en 1776, des réformes de Gribeauval dans l'artillerie avec le ministère Saint-Germain? Ou n'est-ce qu'une simple impatience? Quoi qu'il en soit, Montalembert publia le premier d'une dizaine de volumes sur son système, qu'il appela « fortification perpendiculaire » en 1776, l'année même où le Corps royal du génie fut constitué officiellement, reçut le qualificatif « royal » et devint un véritable corps militaire? 
7 Deux ans plus tard, pendant la guerre d'Amérique, Montalembert fut invité à démontrer ses idées en bâtissant un petit fort sur l'île d'Aix, à l'embouchure de la Charente. Malgré sa promesse de construire le fort en quelques mois, il ne réussit pas à l'achever complètement avant que le projet fût annulé par le ministre en 1783. Tout au long de cet épisode, une véritable guerre éclata entre Montalembert et les ingénieurs des bureaux ministériels. Fourcroy de Ramecourt, devenu le chef du nouveau Corps royal en 1776, était tenu au courant des travaux de l'île d'Aix par ses subordonnés sur place, d'abord Coulomb, envoyé comme représentant du corps à la demande de Montalembert lui-même, puis d'autres ingénieurs qui lui succèdèrent dans cette tâche ingrate, pleine de conflits avec le marquis fortificateur. Montalembert organisa des épreuves pour faire face aux ingénieurs qui prétendaient que le fort, construit en bois, ne supporterait pas les vibrations de la décharge de plus de 100 canons à la fois. Si les représentants du Corps royal du génie lui étaient hostiles, tel n'était pas le cas du représentant du Corps de l'artillerie, Choderlos de Laclos, qui, à l'encontre de Coulomb et de ses collègues, devint un partisan enthousiaste du marquis et de son système.

De retour à Paris dans une espèce de disgrâce - le ministre coupa court à toute correspondance avec lui au sujet du fort de l'île d'Aix et fit la sourde oreille à ses demandes de pension -, Montalembert publia en 1784 son cinquième tome sur la fortification perpendiculaire. Entre autres choses, le livre contient une critique très sévère de Louis de Cormontaigne (1696-1752) - après Vauban, l'ingénieur militaire le plus respecté de Fourcroy et de la quasi-totalité des autres membres du corps - et de Pierre de Filley (1695-1779), autre ingénieur éminent qui termina sa carrière comme général commandant les ingénieurs militaires français en Allemagne pendant la guerre de Sept Ans. Une riposte des ingénieurs était inévitable. Cette fois, elle prit non pas la forme d'un mémoire destiné à être mis sous les yeux du seul ministre, mais celle d'un mémoire lu dans l'auguste forum de l'Académie des sciences.

9 Le 26 février 1785, Fourcroy de Ramecourt lut un mémoire à l'Académie sur les moments de fortification, concept qu'il attribuait - en fait sans aucune preuve - à Vauban. Le moment de fortification est le chiffre obtenu en divisant la durée de la résistance potentielle d'un front de fortification (la partie d'une fortification contenant une courtine et les parties des bastions qui la flanquent) face à une attaque ennemie par le coût de construction d'un tel front. Nombre bizarre que ce quotient d'un nombre précis (coût de construction) et d'un nombre très approximatif basé sur des considérations subjectives sur la performance d'une garnison assiégée.

10 La réponse de l'Académie à cet effort pour faire de la fortification une science quantitative fut mesurée le rapport présente Vauban comme « un de ses membres [...] dont la mémoire lui est respectable et chère» et dont la méthode, fondée sur l'expérience et l'observation, "mérite d'être connue ", car elle peut être utile et "mérite d'être imprimée sous le privilège de l'Académie». Mais la compagnie crut devoir observer en même temps que

« les auteurs du mémoire n'ayant donné que les principes généraux de la méthode et n'ayant rien publié sur les données d'après lesquelles on peut en faire l'application à des cas particuliers, l'académie ne peut juger du degré d'exactitude et de précision des résultats auquel on peut atteindre dans l'état actuel de l'art de construire, de défendre et d'attaquer les places. $»^{8}$

11 Un mois plus tard Fourcroy fut élu associé libre de l'Académie'. Son élection est due davantage à sa position officielle comme chef du Corps du génie qu'à ses talents de savant ou même de technicien. Il avait d'ailleurs été précédé à l'Académie par quelques- 
uns de ses subordonnés plus brillants et beaucoup plus jeunes que lui, Charles-Augustin Coulomb (1736-1806) et Jean-Baptiste Meusnier de la Place (1754-1793), lesquels avaient, de plus, le statut d'académiciens réguliers et non pas associés libres ${ }^{10}$.

La circonspection de l'Académie est bien compréhensible, vu l'histoire de la polémique entre Montalembert et le Corps royal du génie. Quand Montalembert avait présenté son premier volume à l'Académie en 1776, Jean-Baptiste Leroy (1720-1800), physicien laborieux mais guère brillant qui avait eu le malheur de présenter Mesmer à l'Académie, avait été le rapporteur. L'année même du triomphe final du système Gribeauval, qui avait ses partisans et ennemis à l'Académie comme à l'armée, Leroy était sans doute sensible aux dangers pour l'Académie de juger un ouvrage radical qui avait des implications immenses pour le système de fortifications françaises. D'autant plus que l'Académie venait d'attirer des critiques publiques, notamment dans les affaires Marat et Mesmer. Pourtant, le rôle de l'Académie comme juge d'inventions et de projets techniques, surtout quand il s'agissait d'un de ses membres, ne laissait pas d'issue. Le jugement de Leroy avait été pondéré et quelque peu équivoque. Il signalait que les militaires admettaient unanimement que l'attaque dominait la défense dans les fortifications actuelles - situation dangereuse pour laquelle il fallait chercher des remèdes. Les objections de Montalembert contre les bastions étaient donc fondées, « au moins jusqu'à un certain point $»^{11}$. Mais il signalait aussi des objections au système de Montalembert, qu'il n'avait pas eu le temps d'approfondir et sur lesquelles il ne voulait pas prendre de position. Le système de Montalembert «ne nous a pas paru établi sur des preuves assez bien liées pour en démontrer les avantages ». En terminant il approuva néanmoins le travail de vingt ans que Montalembert avait consacré aux fortifications et en recommanda l'approbation par l'Académie.

Jusqu'au cinquième, les tomes suivants de l'ouvrage de Montalembert reçurent ensuite une simple approbation de l'Académie sur la recommandation de Leroy, qui ne donna plus un rapport détaillé comme pour le premier. Mais pour le cinquième, en 1785, Leroy se prononça sur la question de l'artillerie, sujet qui occupait une partie importante du tome dans lequel Montalembert attaquait Cormontaigne et Filley. Cette fois le désaccord de Leroy avec Montalembert fut plus marqué, quoiqu'il ait aussi pris soin de mentionner que, au contraire, les officiers de la marine à l'île d'Aix avaient trouvé bons les canons de Montalembert ${ }^{12}$.

Bien que l'Académie ait à nouveau "approuvé » le travail de son membre Montalembert, ce n'était donc guère avec enthousiasme. L'hypothèse que Leroy et l'Académie aient été sensibles aux opinions des artilleurs et ingénieurs en se prononçant sur le mérite des idées techniques concernant leurs métiers est bien plausible. Dans son sixième tome, présenté à l'Académie moins de deux semaines après que Fourcroy y ait lu son mémoire sur les moments de fortification, Montalembert donne une réfutation des critiques de son système de fortification adressées au ministre de la Guerre pendant la construction du fort de l'île d'Aix par un certain capitaine Grenier du corps du génie. S'il espérait que ce volume lui donnerait un triomphe définitif sur ses ennemis au sein du Corps du génie, il dut être déçu : dans les semaines qui suivirent, Fourcroy obtint la même approbation, aussi mesurée que la sienne il est vrai, pour son propre mémoire, avant d'être reçu comme son confrère à l'Académie.

Un peu plus d'un an plus tard, Fourcroy profita de ce nouveau statut pour lancer contre Montalembert une attaque beaucoup plus violente et quelque peu sournoise. Il publia 
les Mémoires sur la Fortification perpendiculaire, officiellement attribués à "plusieurs officiers du corps royal du génie », mais dont l'auteur était en fait Fourcroy lui-même, peut-être assisté d'un officier subalterne. Présenté à l'Académie le 16 juin 1786, l'ouvrage contenait son mémoire sur les moments de fortification de l'année précédente, augmenté d'une application de ces calculs au système de Montalembert pour démontrer qu'il était nettement inférieur aux systèmes classiques de Vauban et Cormontaigne. Comme Montalembert allait le dire plus tard, l'inclusion de l'approbation de l'Académie donnée au mémoire, beaucoup plus court, que Fourcroy avait lu à l'Académie, pouvait faire croire au lecteur moyen que l'Académie avait approuvé l'ensemble du nouvel ouvrage.

La réponse de Montalembert ne se fit pas attendre. Le 8 juillet 1786, il présenta à son tour une réfutation longue et détaillée du texte de Fourcroy à l'Académie dans son septième tome de la Fortification perpendiculaire ${ }^{13}$. À sa demande formelle, la commission académique nommée pour en rendre compte fut mixte : aux côtés des commissaires qui avaient examiné ses précédents volumes, Leroy et Borda, siègèrent ceux qui venaient d'examiner le mémoire de Fourcroy, Condorcet et Laplace. À la demande de Montalembert lui-même, l'Académie était ainsi érigée en véritable tribunal.

Dès ses débuts, l'Académie a toujours jugé les ouvrages de ses membres, les inventions des inventeurs qui demandaient un « privilège » de l'État, et les mémoires scientifiques. Elle répondait aussi aux questions techniques et d'utilité publique que lui soumettait le gouvernement. La compétence de l'Académie dans les sciences en faisait l'arbitre naturel de ces questions dans la société de l'Ancien Régime. Mais si elle pouvait se cantonner exclusivement au domaine scientifique quand il s'agissait des mémoires des savants, la nature même de ces questions techniques l'entraînait inévitablement dans les domaines administratifs, voire politiques : la fortification était affaire d'État, et de la plus haute importance, avec des implications d'ordre budgétaire et administratif. Dans l'affaire Marat, l'Académie traçait la ligne de partage entre la pseudoscience charlatanisme était le mot à la mode - et la vraie science. C'était bien sa fonction officielle. Mais dans la polémique entre les ingénieurs et Montalembert, des doutes sur le rôle de l'Académie pourraient surgir. Pour les corps savants de l'armée française du dix-huitième siècle, le génie et l'artillerie, il fallait bien être savant. C'était là un aspect important de leur légitimité et de leur statut distinctif parmi les autres corps militaires. Mais, pour juger des questions les concernant, il fallait aussi l'expérience de la guerre et des connaissances particulières des objets, matières, procédés et habitudes de leur métier. Le Corps du génie prétendait ainsi, dans son domaine, à un rôle d'arbitre équivalent à celui de l'Académie pour les sciences, d'autant plus qu'il venait enfin d'obtenir, par de longues et difficiles luttes, une identité professionnelle. En faisant appel à l'Académie, Montalembert contestait donc ouvertement le droit des ingénieurs à juger de leurs propres affaires. Il offrait à l'Académie l'empire sur toutes les activités scientifiques et techniques. Condorcet, son secrétaire perpétuel, n'était en fait guère enclin à accepter complètement un tel rôle.

Montalembert lui-même n'avait pas toujours agi ainsi. En 1761, quand il avait présenté pour la première fois le prospectus de son nouveau système à Choiseul, il faisait appel au public, « ce grand appréciateur de toute production », mais il ajoutait aussi, de façon un peu contradictoire : «Au reste, c'est aux gens du métier à juger si les tentatives que j'ai faites pour ouvrir une nouvelle carrière, peuvent produire quelques changemens avantageux... $»^{14}$ - Fourcroy, appelé à donner son avis à l'époque, n'avait pas manqué de 
souligner ce passage. "La valeur est pour ainsi dire innée dans le corps des ingénieurs", déclarait encore Montalembert dans l'Avant-Propos de son premier volume, en $1776^{15}$. Le ton a vite changé. Dans les années 1780 , ce sont d'abord les directeurs du génie (les chefs des circonscriptions territoriales du corps du génie) qui sont accusés de faire pression sur le chef à Versailles pour empêcher l'adoption du système de Montalembert ; puis c'est ce chef lui-même, Fourcroy, qui, par sa puissance dictatoriale, se fait « l'éteignoir » des nouvelles idées ${ }^{16}$.

19 En 1784, Montalembert attaque les ingénieurs comme un corps exclusivement intéressé à garder ses privilèges - thème qui commence à devenir assez banal à cette époque. Il compare les ingénieurs à un corps imaginaire d'explorateurs, qui resteraient chez eux payés par l'État pour voyager et faire des découvertes et qui ne prendraient pas la mer, sous prétexte qu'il n'y a plus de découvertes à faire et que tout serait déjà découvert. Ils rejetteraient ainsi les découvertes d'un capitaine Cook et s'abriteraient derrière leur statut privilégié pour ne pas être obligés de faire de rudes voyages, loin de leur vie confortable à terre. Mais toutes les corporations ne sont pas mauvaises.

«Il n'en est pas de même des célébres compagnies savantes, établies dans les différentes États de l'Europe, et nommément celle dont j'ai l'honneur de faire partie. Toujours, en activité, toujours employant sans relâche, les profondes connaissances de chacun de ses membres, elle ne cesse de faire des découvertes et d'applaudir à celles qui se font, de quelque part qu'elles viennent. Les vrais savants, les grands génies, vivent en sécurité au milieu des savants; on ne peut ôter un mérite réel. On en sent en soi l'existence, et l'on ne craint point celui des autres ; c'est un empire si justement acquis, que celui dont jouit cette illustre Compagnie, qu'elle ne craint point de le perdre, et loin de chercher à obscurcir l'éclat des nouveaux talents, elle ne manque jamais de les protéger. L'effet naturel du savoir est d'aimer ceux qui cultivent la science ; les faibles ont seuls la crainte en partage, ils sont naturellement ombrageux; l'envie et la jalousie sont toujours le caractère des petites âmes. $»^{17}$

20 Un an plus tard, cette Académie si noble et exempte des vices du corporatisme risque de se déshonorer en accueillant en son sein Fourcroy, le chef de ses ennemis et celui d'une corporation rétrograde. Montalembert mise toujours sur l'appui de l'Académie. Tout en se moquant des ingénieurs, il continue de la louer dans son tome 7 , la riposte à Fourcroy :

"L'assurance des auteurs de cette méthode [les moments de fortification de Fourcroy], en la représentant à une Académie où il règne autant de lumière que de sagesse, fait présumer qu'ils comptent obtenir d'elle une confiance aveugle sur une matière qu'ils croient devoir lui être étrangère. Ils se sont rendus prolixes et diffus, espérant que leur obscurité ne serait attribuée qu'à la nature de la question à décider, et non à leur style : mais ils ne connaissent pas l'Académie ; les principes d'aucun art ne lui sont inconnus; toutes les sciences y trouveraient des professeurs; et la science militaire ne saurait manquer de Juges instruits dans toutes ses parties. D'ailleurs, les assertions de leur mémoire sont si peu admissibles, qu'avec la justesse d'esprit et l'habitude de l'analyse répandue si généralement parmi tous ses membres, il n'y en a pas un seul qui puisse prendre pour des vérités des erreurs répandues avec tant de profusion. C'est donc à son tribunal que j'en appelle, des décisions prononcées contre l'ouvrage que j'ai publié depuis dix ans, avec son approbation. $»^{18}$

Le respect affiché pour ses confrères de l'Académie n'empêche pas son discours de devenir de plus en plus violent. Le ministre de la Guerre lui ordonne de retrancher de son livre certaines phrases, considérées comme injurieuses pour Fourcroy. Il est clair 
que l'Académie se sent gênée. Condorcet écrit dans le procès-verbal du 23 décembre 1786 :

«J'ai demandé si dans le cas où l'on consulterait l'Académie sur la publication d'un mémoire contre un de ses membres, elle devait examiner ce mémoire et répondre d'après cet examen ; il a été unanimement décidé qu'elle ne devait pas s'en mêler en aucune manière. $»^{19}$

Ce n'est que près de deux ans après avoir soumis son livre au jugement de l'Académie que Montalembert en reçut la réponse. Elle était loin d'être l'approbation inconditionnelle qu'il attendait. Condorcet, Borda, Laplace et Leroy signèrent le rapport suivant :

«On concevra sans peine que pour faire valoir les raisons qu'il oppose à MM. les officers du Génie, M. de Montalembert a été obligé de s'étendre sur les différents objets de cette discussion, c'est aussi ce qui est arrivé. Par là il serait impossible de le suivre dans tout ce qu'il dit sur ce sujet important, sans se jeter dans des explications et des détails trop longs, et trop difficiles à saisir.

Ainsi nous nous contenterons d'observer que M. de Montalembert nous a paru discuter avec détail dans sa réponse, plusieurs points importants de l'art de la fortification, et particulièrement ceux qui regardent la manière de juger de la force absolue, et de la valeur réelle des places de guerre. Que pour mieux répondre à MM. les officiers du Génie, il y a développé davantage plusieurs articles sur lesquels il ne s'était pas assez étendu dans son traité de la Fortification perpendiculaire. Enfin que cette réponse ne peut que répandre du jour sur la dispute importante qui s'est élevée depuis quelque temps sur l'infériorité de l'art de la défense des places, par rapport à celui de l'attaque.

Nous croyons en conséquence que la réponse de $\mathrm{M}$. le Marquis de Montalembert, qui peut faire d'ailleurs suite à son ouvrage, mérite d'être imprimée sous le privilège de l'Académie, sans prendre d'ailleurs aucun parti sur le fonds de la question. ${ }^{20}$

Le plumitif nous informe que la dernière phrase du rapport avait été rectifiée: la formulation initiale («mérite d'être approuvé par l'Académie comme cet ouvrage l'a été ») a été biffée et Condorcet a ajouté in fine la prudente réserve ("sans prendre d'ailleurs aucun parti sur le fonds de la question»).

Publiquement, Montalembert afficha son contentement. Il a même reproduit le rapport de l'Académie dans l'Avant-Propos de la version finale imprimée de son livre et a ajouté une exégèse quelque peu laborieuse pour montrer que les académiciens lui donnaient raison contre Fourcroy. Mais il paraît bien qu'il fut touché à vif. Premier signe : la publication, au début de la version définitive de son livre, d'une lettre de Lazare Carnot louant le système de Montalembert en termes enflés avec, selon Marcel Reinhard, la "verve caustique [qui] est bien celle d'un prérévolutionnaire, d'un disciple des philosophes, d'un contemporain de Beaumarchais... ${ }^{21}$. Carnot écrivit que le livre de Montalembert :

« [...] paraissait à la vérité sous le nom d'un officier général et d'un membre de l'Académie des Sciences; mais comme ces titres n'annoncent ordinairement guère plus de mérite réel dans un homme de qualité, que ceux de duc ou de cordon-bleu, ce ne pouvait être une raison bien forte, ignorant alors que vous faisiez une exception si honorable pour vous et pour les Corps célèbres dont vous êtes membre. $»^{22}$

Voilà qui était un peu fort de la part d'un jeune officier du génie qui avait reçu une mention honorable de l'Académie pour son Essai sur les machines en 1781 et dans un livre que l'Académie jugeait digne d'être imprimé. 

décidé à ne pas battre en bréche la forteresse de l'Académie, mais plutôt à y faire un travail de sape. En février 1787 parut un pamphlet assez long sous la signature de son cousin éloigné, Jean-Charles, baron de Montalembert ${ }^{23}$. En principe, il s'agissait d'une réponse au compte rendu favorable du livre de Fourcroy, les Mémoires sur la Fortification perpendiculaire, par plusieurs officiers du génie, fait par le chevalier Kéralio dans le Journal de Savants.

S' cest le baron qui signe la lettre ouverte dans le pamphlet, le style et le ton font songer au marquis. On y retrouve les mêmes arguments prolixes et ennuyeux attaquant les ingénieurs et louant le marquis. Mais ce qui attire l'attention, c'est surtout une attaque féroce contre l'Académie des sciences. Sans pouvoir le prouver, je suis convaincu que le marquis de Montalembert lui-même en est l'auteur ou l'inspirateur principal. Montalembert a d'ailleurs réimprimé ce pamphlet, sans commentaire, dans le tome 9 de la Fortification perpendiculaire, paru en 1790, après la disparition de la censure royale.

Revenons au baron. Il se dit non retenu par les scrupules sur la bienséance qui sont si caractéristiques de son cousin offensé. Mais, ami de la vérité, il est obligé de la défendre, même si la délicatesse de son cousin l'empêche de le faire. La description de la vie ordinaire de l'Académie fait songer aux attaques de Mesmer.

«Il arrive assez souvent, à la plupart de ces messieurs, de causer, ou écrire leurs lettres pendant les rapports, et ils n'en sont pas moins de l'avis de celui qui a parlé, surtout lorsque c'est un des faiseurs de l'Académie; et M. de Condorcet, que l'on sait avoir été le principal auteur du rapport, était accoutumé à n'y point trouver de contradicteurs. $»^{24}$

Le rapport en question est celui que les commisaires de l'Académie avaient rendu sur le premier mémoire de Fourcroy, en mars 1785. Le péché de l'Académie, c'est d'avoir approuvé un mémoire par

« [...] M. Fourcroy, directeur des fortifications, dans la faveur du ministre, et résidant auprès de lui ; il fallait le contenter, pour plaire à son protecteur. Les membres de cette Académie se sont donc montrés dans cette occasion (et ce n'est pas la seule) meilleurs courtisans que bon philosophes. Qu'a-t-il pu résulter de pareilles dispositions? Un jugement, où la partialité des juges a suppléé à leur manque de connaissances; étant évident que MM. les commissaires n'en avaient aucune de relative à ce qu'ils avaient à décider. Cette Compagnie, savante dans tant d'autres parties, n'est instruite de rien de ce qui concerne l'art militaire. M. De la Place, examinateur des Écoles d'Artillerie, était le seul dans les trois commissaires qui fut en état d'avoir un avis fondé sur ses lumières; mais il n'avait que sa voix contre deux : on sait que tout ce qu'il a pu faire, c'est de mitiger le rapport [...] $\gg^{25}$

Ces paroles officiellement signées du baron de Montalembert, colonel avec une souslieutenance dans les chevau-légers de la Garde du roi, ne sont pas éloignées de celles de Marat, auteur des Charlatans modernes. Selon le baron, les académiciens sont serviles, égoïstes et attachés à leur privilèges. De plus, ils ne savent pas de quoi ils parlent quand il s'agit des affaires militaires. Le marquis affirmait le contraire trois ans plus tôt, quand il faisait appel aux lumières de l'Académie contre l'attitude bornée des ingénieurs. Une véritable révolution s'est donc opérée dans l'esprit des Montalembert.

31 Pour conclure, il convient de comparer très brièvement le cas Montalembert à ceux de Mesmer et Marat.

Annales historiques de la Révolution française, 320 | avril-juin 2000 

de l'Académie à ses idées, bien qu'il n'ait jamais subi le rejet brutal qui a été le sort des premiers. Il se trompait quand il disait, en 1784, que les savants de l'Académie applaudissaient aux découvertes, «de quelque part qu'elles viennent». À propos du mesmérisme, Condorcet avait écrit :

«Mais le magnétisme animal a été admiré, employé par des physiciens ou des médecins. J'en conviens, mais il s'agit de me déterminer à croire sur une autorité ; cela est dur pour la raison humaine. Ainsi je n'entends point par physicien ou par médecin un homme qui a fait des livres de physique ou qui a été reçu docteur dans quelque faculté. J'entends un homme qui, avant qu'il fût question du magnétisme, jouissait en France, en Europe même, d'une réputation bien établie. Voilà l'espèce de témoignage qu'il me faut pour croire un fait extraordinaire de physique ou de médecine. $»^{26}$

À une époque où la science était en train de devenir une véritable profession, Marat et Montalembert avaient tous deux oublié l'importance de l'autorité dans la République des Sciences, même si elle affichait publiquement son mépris pour les autorités. Condorcet fut non seulement solidaire de la Société royale de médecine contre Mesmer, mais aussi du Corps royal du génie contre Montalembert, bien qu'il ait probablement eu des doutes sur la compétence de Fourcroy dans ses analyses du système de Montalembert.

Ni Montalembert ni Mesmer n'acceptèrent leur échec à l'Académie. Ils eurent recours au forum public. Compréhensible chez un Mesmer ou un Marat, cette attitude constituait un grave manquement à la bienséance dans le cas de Montalembert, luimême académicien. Dans les deux cas, un autre corps savant - la Société royale de Médecine et le Corps royal du génie - était mis en cause, et, dans les deux cas, l'Académie fut solidaire de ces corps officiels, démontrant ainsi que le professionnalisme avait besoin d'autorité, en dépit de l'idéologie affichée.

discours prérévolutionnaire semble un peu étrange dans la bouche d'un noble et membre de la garde du roi. On en entendait pourtant ailleurs que dans les sciences: l'Assemblée des notables s'est réunie dans une atmosphère frondeuse en février 1787. Je terminerai mon essai en réfléchissant sur cet événement. La grande différence entre Montalembert et un Marat ou un Mesmer, c'est que le premier est membre de l'Académie ; il agit donc, non de l'extérieur, mais au sein même de l'institution. C'est un noble, un «notable » de l'Ancien Régime; comme les autres notables de la société en général, il rechigne à se soumettre à l'autorité. Il comprend mal la nature et l'importance de l'autorité dans le monde des savants. Tout comme l'aristocratie française a joué un certain rôle dans l'avènement de la Révolution et la chute du pouvoir royal, ce notable de la science a fait partie du mouvement de contestation du pouvoir académique. Comme eux, et à la différence de Marat, il l'a fait de l'intérieur de l'institution et non de l'extérieur. La noblesse perdra son rôle privilégié officiel pendant la Révolution; l'Académie, ressuscitée sous le Directoire, perdra aussi ses membres honoraires et associés libres. Il n'y aura désormais place que pour des savants de plus en plus professionnels. Montalembert sera parmi ceux qui en feront les frais. Il ne sera pas choisi pour faire partie de l'Institut national en 1795 et quand il s'y présentera comme candidat à la place de Carnot, «fructidorisé » en 1797, il ne sera pas élu. Il est vrai que le vainqueur de cette élection, un certain Bonaparte, pourrait bien être considéré comme une sorte de membre honoraire ou d'associé libre de l'Institut. Mais il sera le dernier de cette espèce dans l'Académie issue de la Révolution. 


\section{NOTES}

1.Sur l'Académie voir Roger Hahn, The Anatomy of a Scientific Institution: The Paris Academy of Sciences, 1666-1803, Berkeley, University of California Press, 1971 et Charles C. Gillispie, Science and Polity in France at the End of the Old Regime, Princeton, Princeton University Press, 1980.

2.Voir son discours du 7 vendémiaire an III (28 septembre 1794) à la Convention, fondant l'École centrale des travaux publics (Polytechnique).

3.Gillispie, op.cit., pp. 81-99.

4.Ibid., p. 283.

5.Nous sommes obligés ici de passer outre les détails de ce système de fortification. On peut en trouver des descriptions dans E.M. Lloyd, Vauban, Montalembert, Carnot : Engineer Studies, Londres, 1887, et chez certains ingénieurs militaires français du XIXe siècle, comme le général Prévost de Vernois, De la fortification depuis Vauban ou Examen des principales innovations qui s'y sont introduites depuis la mort de ce grand homme, 2 vol., Paris, Dumaine, 1861, et Cosseron De Villenoisy, Essai historique sur la fortification, Paris, Dumaine, 1869, qui sont en général hostiles aux idées de Montalembert. Simon-François Gayvernon (Traité élémentaire d'art militaire et de fortification, à l'usage des élèves de l'École polytechnique, et des élèves des Écoles militaires, 2 vol., Paris, Allais, an XIII [1805]), professeur de fortification à l'École polytechnique pendant la Révolution et l'Empire, et C.-F. Mandar (Architecture des forteresses, ou De l'Art de fortifier les places, et de disposer les Établissemens de tout genre, qui ont rapport à la guerre, Paris, Magimel, 1801), ancien dessinateur de Montalembert, en ont aussi donné des descriptions.

6.Il ne faut pas confondre l'ingénieur militaire Charles-René de Fourcroy de Ramecourt (1715-1791) avec son cousin éloigné mentionné plus haut, le chimiste Antoine-François Fourcroy (1755-1809).

7.Marc-René, marquis de Montalembert, La Fortification perpendiculaire, ou Essai sur plusieurs manières de fortifier la ligne droite, le triangle, le carré, et tous les polygônes, de quelqu'étendue qu'en soient les côtés, en donnant à leur défense une direction perpendiculaire. où l'on trouve des méthodes d'améliorer les places déjà construites, et de les rendre beaucoup plus fortes. On y trouve aussi des Redoutes, des Forts et des Retranchemens de campagne, d'une construction nouvelle, Paris, Philippe Denys-Pierres, 1776. Montalembert publia onze volumes sur la fortification perpendiculaire jusqu'à sa mort. Il y eut une réimpression pendant la Révolution.

8.Rapport de l'Académie sur le mémoire de Fourcroy, Académie des sciences, Procèsverbaux, 16 mars 1785. Texte reproduit par Montalembert dans la Fortification perpendiculaire, t. 7, Avant-Propos, p. 14.

9.Le 23 avril 1785.

10.Coulomb fut élu membre correspondant en 1773 et mécanicien adjoint en 1781; Meusnier était membre correspondant depuis 1776 et géomètre adjoint depuis 1784. 11.Extrait des procès-verbaux de l'Académie du 13 mars 1776, dossier Montalembert, Académie des sciences.

12.Montalembert avait présenté un nouveau modèle d'affût, qu'il avait construit pour les canons du fort de l'île d'Aix. Pour des raisons différentes, les deux camps d'artilleurs dans la querelle des « rouges » et des « bleus » - les partisans de Vallière et de Gribeauval - s'entendaient pour rejeter les idées de Montalembert sur l'artillerie. 
13.Ce septième volume porte le titre Réponse au Mémoire sur la Fortification Perpendiculaire par plusieurs officiers du Corps Royal du Génie, Présenté à l'Académie Royale des Sciences, Paris, Philippe-Denys Pierres, 1787.

14.Montalembert, « Discours préliminaire », p. 15, de son prospectus présenté au Ministre, Service historique de l'armée de terre, Vincennes, Archives du Génie, Art.21 sect. 1 \$1 cart.5.

15.Fortification perpendiculaire, t. 1, « Avant-propos », p. xx.

16.Le mot et l'insulte sont de Carnot, mais repris par Montalembert.

17.Fortification perpendiculaire, t. 5, « Avant-Propos », pp. v-vi.

18.Ibid., t. 7, pp. 310-311.

19.Procès-verbaux de l'Académie des sciences, le 23 décembre 1786.

20.Procès-verbaux de l'Académie des sciences, le 12 avril 1788.

21.Marcel Reinhard, Le Grand Carnot, 2 vol., Paris, Hachette, 1950-1952, t. 1, p.113. Nous ne pouvons pas entrer ici dans l'histoire extrêmement intéressante des relations entre Lazare Carnot et Montalembert, décrites par Reinhard dans le chapitre 8 de son ouvrage magistral. Il suffit de dire que Carnot a été le seul officier du génie pendant l'Ancien Régime à afficher publiquement son appui aux idées de Montalembert, et cela bien qu'il ait été auparavant malmené anonymement par le marquis à propos de l'Éloge de Vauban qu'il avait écrit ! Tout au long de la polémique à l'Académie des sciences, Montalembert et son ami Choderlos de Laclos publièrent des attaques contre les fortifications de Vauban qui eurent un grand retentissement à l'armée et dans le public. 22.Lettre de Carnot à Montalembert du 21 août 1788, reproduite dans Montalembert, Réponse..., « Avertissement ", pp. 11-12.

23.Jean-Charles de Montalembert, Lettre de M. le Baron de Montalembert à M. de Keralio, en Réponse au compte qu'il a rendu dans le Journal des Savants, du Mémoire sur la Fortification perpendiculaire, publié sous le nom de plusieurs officers du Corps Royal du Génie, en 1786, London, T. Spilsbury et Fils, s.d. Le lieu de publication fait penser que le pamphlet pourrait avoir été publié sous le manteau. Réimprimé dans la Fortification perpendiculaire, t. 9, Paris, 1790.

24.Ibid., p. 28.

25.Ibid., p. 28.

26.Manuscrit écrit vers la fin 1784 ou en 1785 , Bibliothèque de l'Institut de France, ms 883, fol. 231-247, cité par Robert Darnton, Mesmerism and the End of Enlightenment in France, 1968, réimpression New York, Schocken Books, 1970, p. 189 (traduction française, Paris, Perrin, 1984).

\section{RÉSUMÉS}

Dans la décennie qui précéda la Révolution, les attaques contre l'Académie royale des sciences se sont multipliées de la part de détracteurs qui, tels Marat ou Mesmer, la dépeignaient comme un repaire d'injustes privilèges fermé aux idées nouvelles. Ces attaques, extérieures à l'Académie, provenaient de personnes auxquelles elle avait refusé reconnaissance et siège. Cette communication décrit une autre campagne, sans doute plus discrète mais tout aussi féroce, 
menée par un de ses membres, le marquis Marc-René de Montalembert (1714-1800), dont l'élection comme associé libre avait été le résultat de ses liens avec la Cour. Soldat et diplomate, Montalembert était auteur d'un système de fortification rejeté avec virulence par la hiérarchie du corps royal du Génie. Dans cette polémique sur la fortification, les deux parties en appelèrent à l'Académie et, lorsque cette dernière refusa de soutenir son système, Montalembert commença à l'attaquer. Le personnage est présenté ici comme le dernier représentant d'un type d'amateur noble qui, n'obtenant pas de ses confrères la déférence qu'il croyait lui être due, rejoignit le discours anti-académique de critiques socialement inférieurs. Ce faisant, il se comporta comme ceux de son ordre qui, dans le domaine politique, attaquèrent la monarchie dans les dernières années de l'Ancien Régime.

Pre-revolutionary discourse at the Academy of Science : the example of Montalembert. In the decade before the French Revolution, the Royal Academy of Sciences came under increasing attack from critics such as Marat and Mesmer. It was depicted by them as closed to new ideas and a den of unwarranted privilege. These attacks were by outsiders who were thwarted in their efforts to gain recognition and admittance to the Academy. This paper describes what appears to be a more discreet but equally ferocious campaign against the Academy by one of the members of the Academy itself, the marquis Marc-René de Montalembert (1714-1800), who was an associé libre of the Academy mainly as the result of court connections. Montalembert was a soldier, diplomat, and author of a system of fortification vigorously rejected by the leadership of the military engineering corps. Both sides in this polemic over fortification resorted to the Academy as a tribunal in their quarrel, and Montalembert began his campaign against the Academy after he failed to win its unqualified support for his system. The paper concludes with the suggestion that he represents the last of a type of noble amateur who expected deference from his fellow academicians and when he did not get it joined his social inferiors in their anti-academic discourse. In this, he behaved like some other members of his class in the domain of politics in general in their attacks on the monarchy in the waning years of the Ancien Régime.

\section{INDEX}

Mots-clés : Académie des sciences, Montalembert, fortification, Fourcroy de Ramecourt, île d'Aix

\section{AUTEUR}

JÂNIS LANGINS

Associate Professor of History of Science and Director. Institute for the History and Philosophy of Science and Technology 